\title{
INTRATHECAL MORPHINE WITH OR WITHOUT TAP BLOCK FOR IMPROVED POSTOPERATIVE PAIN RELIEF IN MAJOR ELECTIVE LAPAROSCOPIC UROLOGICAL SURGERY
}

\author{
S. Biuk ${ }^{1}$, K. Krishnan ${ }^{1}$, W. Allison ${ }^{1}$ \\ ${ }^{1}$ Northern Lincolnshire and Goole NHS Foundation Trust, Anaesthetics, Scunthorpe, United Kingdom
}

\section{Background and Aims:}

Adequate postoperative pain relief is key to early recovery. The use of intrathecal morphine (IT morphine) in addition to a general anaesthetic has become increasingly common practice.

The use of a Transversus Abdominal Plane block (TAP block) administered under direct vision by the surgeon is also thought to improve postoperative pain relief and reduce opiate requirement.

The aim of our study was to determine if the addition of a TAP block did indeed improve postoperative outcomes.

\section{Methods:}

We did a retrospective review of all major elective laparoscopic urological surgeries dating as far back as December 2014. We looked at all the procedures in which IT morphine was administered at the time of induction of anaesthesia. Cases that had missing data, or for which the clinical notes were not obtained were excluded from the study.

The patients were divided in to two groups:

1) Patients who received IT morphine as well as a TAP block.

2) Patients who received IT morphine without a TAP block.

The two groups were then compared in regards to the following:

a) VAS Pain scores up to 48 hours postoperative.

b) Amount of IV PCA morphine/fentanyl used up to 48 hours postoperative.

c) Patient demographics (gender, age).

d) Duration of surgery.

e) Average dose of regional anaesthesia and other analgesia administered both intraoperatively and also in the 48 hour postoperative period.

f) Duration of hospital stay.

g) Occurrence of side-effects: Respiratory depression, Hypoxia, Sedation, Hypotension, Oliguria, Pruritis and Urine retention.

h) Time to commencement of oral intake (Fluids \& Foods).

i) Time to first mobilisation.

\begin{tabular}{|c|c|c|c|c|c|c|c|c|}
\hline \multirow{2}{*}{ Oral intake } & \multicolumn{2}{|c|}{ Day 0 } & \multicolumn{2}{c|}{ Day 1 } & \multicolumn{2}{c|}{ Day 2 } & \multicolumn{2}{c|}{ Day 3 } \\
\cline { 2 - 9 } & Fluid & Food & Fluid & Food & Fluid & Food & Fluid & Food \\
\hline TAP & $40 \%$ & $4 \%$ & $52 \%$ & $68 \%$ & $4 \%$ & $12 \%$ & $0 \%$ & $0 \%$ \\
\hline No TAP & $74 \%$ & $23 \%$ & $23 \%$ & $49 \%$ & $3 \%$ & $5 \%$ & $0 \%$ & $0 \%$ \\
\hline
\end{tabular}

\begin{tabular}{|c|c|c|c|c|c|c|}
\hline Mobilisation & Day 0 & Day 1 & Day 2 & Day 3 & Day 4 & Day 5 \\
\hline TAP & $0 \%$ & $48 \%$ & $40 \%$ & $4 \%$ & $4 \%$ & $0 \%$ \\
\hline No TAP & $0 \%$ & $67 \%$ & $18 \%$ & $0 \%$ & $0 \%$ & $3 \%$ \\
\hline
\end{tabular}

Results:

The patients who received IT morphine alone had lower pain scores and less use of IV PCA morphine than the patients who received IT morphine with TAP block $(\mathrm{P}=0.00124$ and $\mathrm{P}=0.04036)$.

Looking at the tables below, it can be noted that both groups were fairly similar in demographics. The average duration of surgery was 0.7 hours longer in the TAP group. The average dose of IT morphine was fairly similar. However, the doses of intraoperative analgesia was quite variable between the two groups. The overall use of postoperative Oramorph was notably higher in the No TAP group.

In regards to side effects, patients in the TAP group were noted to have more reduced sedation scores and oliguria, where as patients in the No TAP group had increased episodes of hypotension, pruritis and urine retention.

Duration of postoperative hospital stay was found to be nearly 3 days longer for patients in the TAP group.

\section{Conclusions:}

Our study demonstrates that the addition of a TAP block does not add any benefit in terms of postoperative VAS pain scores or use of IV PCA morphine. However, it does lead to a reduction in the use of other postoperative analgesics, particularly Oramorph.

\begin{tabular}{|l|c|c|}
\hline & TAP & No TAP \\
\hline Male:Female (\%:\%) & $15: 10(60: 40)$ & $26: 13(67: 33)$ \\
\hline Avg* Age (range) & $65.4(34-84)$ & $65.4(28-87)$ \\
\hline Avg Procedure Length (hrs) & 4.1 & 3.4 \\
\hline Avg IT Morphine (mcg) & 316 & 340 \\
\hline Avg 0.5\% Bupivacaine (mg) & 5 & 10 \\
\hline Avg IO Fentanyl (mcg) & 120 & 215 \\
\hline Avg IO Morphine (mg) & 8.92 & 2.5 \\
\hline$\%$ received IO Paracetamol & $88.0 \%$ & $72.0 \%$ \\
\hline$\%$ received IO NSAIDS & $12.0 \%$ & $15.4 \%$ \\
\hline \% requiring IO inotropes & $60.0 \%$ & $53.8 \%$ \\
\hline Avg PO Paracetamol (gm) & 6.28 & 7.58 \\
\hline$\%$ requiring PO NSAIDS & $4.0 \%$ & $10.3 \%$ \\
\hline \% requiring other PO analgesia & $72.0 \%$ & $46.2 \%$ \\
\hline Avg Oramorph at 0-6hrs (mg) & 0.4 & 0.5 \\
\hline Avg Oramorph at 6-12 hrs (mg) & 2 & 0.9 \\
\hline Avg Oramorph at 12-24 hrs (mg) & 3.2 & 7.4 \\
\hline$\%$ using IV PCA Morphine/Fentanyl & $44.0 \%$ & $28.2 \%$ \\
\hline Lowest PO Respiratory Rate (RR) & 13 & 11 \\
\hline Avg time of lowest RR (hrs) & 12.9 & 6 \\
\hline Avg lowest O2 Saturation & $95.0 \%$ & $94.0 \%$ \\
\hline$\%$ with reduced PO sedation score & $28.0 \%$ & $5.1 \%$ \\
\hline Avg IO IV fluids (Litres) & 2.6 & 2.4 \\
\hline Avg PO IV fluids (Litres) & 1.9 & 2.7 \\
\hline$\%$ with episode(s) PO Hypotension & $12.0 \%$ & $12.8 \%$ \\
\hline$\%$ with episode(s) PO Oliguria & $12.0 \%$ & $2.6 \%$ \\
\hline$\%$ with PO pruritis & $20.0 \%$ & $30.8 \%$ \\
\hline$\%$ with urine retention & $0.0 \%$ & $2.6 \%$ \\
\hline Avg Length of Hospital Stay (Days) & 7.64 & 4.92 \\
\hline & & \\
\hline & & \\
\hline
\end{tabular}

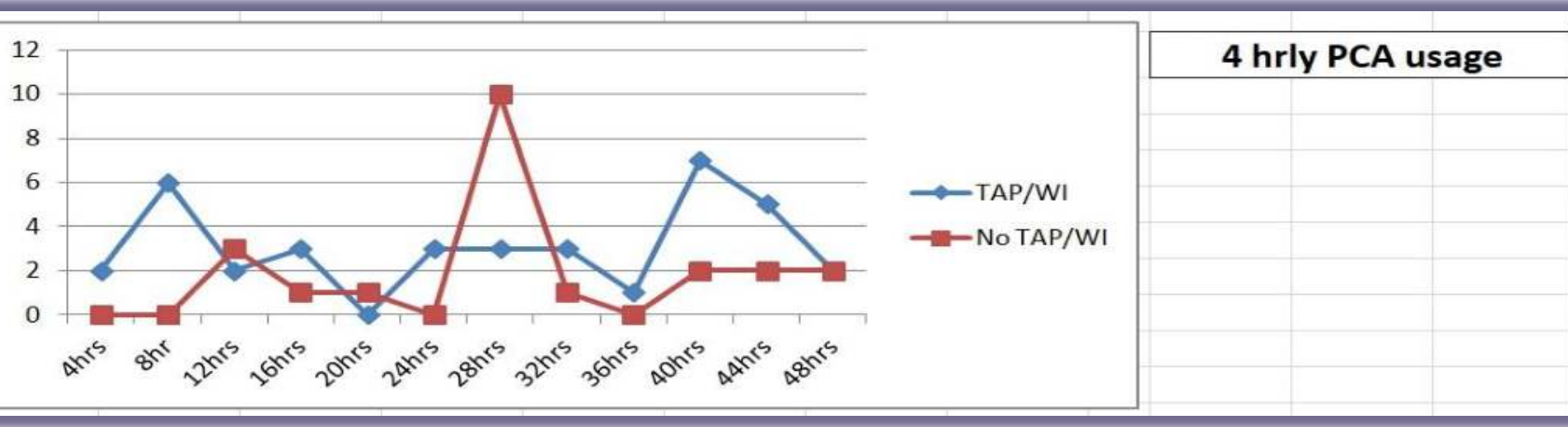

